\title{
Coronavirus Disease Pandemia 2019: Growth of Epidemic Dangers
}

\section{Vo Shapovalova ${ }^{1}$, SI Zbrozhek ${ }^{1}$, EV Suprun ${ }^{1}$, VV Shapovalov ${ }^{1-4 *}$ and VV Shapovalov ${ }^{5}$}

${ }^{1}$ Kharkiv Medical Academy of Postgraduate Education, Kharkiv, Ukraine

${ }^{2}$ Ukrainian National Bar Association, Kyiv, Ukraine

${ }^{3}$ Advocates Company "Apotheosis", Kharkiv, Ukraine

${ }^{4}$ Public Organization "Association of Medical and Pharmaceutical Law", Kharkiv,

Ukraine

${ }^{5}$ Healthcare Department of Kharkiv Regional State Administration, Kharkiv,

Ukraine

*Corresponding Author: VV Shapovalov, Kharkiv Medical Academy of

Postgraduate Education, Kharkiv, Ukraine.
Received: June 02, 2020

Published: July 01, 2020

(C) All rights are reserved by VV hapovalov., et al.

\begin{abstract}
The article is devoted to the problem of the emergence in 2019 of diseases caused by the new coronavirus ("coronavirus disease 2019”), which have already gone down in history as an emergency of international importance. The most common clinical manifestation of a new infection is pneumonia, and in a significant proportion of patients - respiratory distress syndrome. A virological, epidemiological and clinical study of severe acute respiratory syndrome, Middle East respiratory syndrome and the outbreak of acute respiratory diseasein 2019-2020 was compared in Wuhan (Hubei, People's Republic of China, China). Analysis of the scientific literature data on the coronavirus disease pandemic 2019 based on the systematization, synthesis and comparison of documentary data regarding: historical aspects of the study of coronaviruses; severe acute respiratory syndrome; Middle Eastern Respiratory Syndrome; SARS-CoV-2 virus disease COVID-19; comparative characteristics of SARS/MERS/COVID-19. When conducting the study, we used the current legislative, regulatory and instructive documents, Internet resources. They used regulatory, documentary, bibliographic, comparative and graphical methods of analysis. The article presents the results of a comparison of the virological, epidemiological and clinical studies of severe acute respiratory syndrome, Middle East respiratory syndrome and the outbreak of acute respiratory disease in 2019-2020 years in Wuhan (Hubei, People's Republic of China, China). Established that the etiological agent of COVID-19 is coronavirus, phylogenetically close to the causative agent of SARS (SARS-related human CoV virus) and related coronaviruses isolated from bats (SARS-related bat $\mathrm{CoV}$ viruses). The data obtained are the basis for the need to continue further research and reviews of the coronavirus pandemic in the direction of finding methods for effective treatment of patients with this pathology. Keywords: Coronavirus; Epidemic Hazard; Pandemic; Severe Acute Respiratory Syndrome (SARS); Middle East Respiratory Syndrome (MERS); COVID-19 Diseases Caused by the SARS-CoV-2 Virus
\end{abstract}

\section{Abbreviations}

SARS: Severe Acute Respiratory Syndrome; MERS: Middle East Respiratory Syndrome; IBV: Murine Hepatitis Virus; ACoV: Avian
Coronavirus; TGEV: Transmissible Gastroenteritis Virus; MCoV: Murine Coronavirus; PhCoV: Pheasant Coronavirus; TCoV: Turkey 
Coronavirus; MHV: Mouse Hepatitis Virus; ACE2: AngiotensinConverting Enzyme 2

\section{Introduction}

According to the ongoing outbreak of a new coronavirus infection in China, the study of the role of coronaviruses in the occurrence of massive infectious diseases and epidemics is becoming particularly relevant.

Coronaviruses (Coronaviridae, CoVs) are a large family of RNA viruses (includes two subfamilies, five genera, 39 species) that can cause acute respiratory infections among humans.

Coronaviruses can affect the respiratory, gastrointestinal tract, liver and central nervous system of humans and many other types of vertebrates, including domestic animals and livestock, birds, bats, etc [1].

Before epidemic outbreaks in 2002 and in 2012, coronaviruses were not considered highly pathogenic for humans, since viruses that previously circulated in the human population in immunocompetent persons mainly caused only mild forms of the disease.

Severe, often lethal forms of pneumonia that occurred during outbreaks of SARS and MERS in individuals without immunodeficiency, made a fresh assessment of the pathogenicity of coronaviruses for humans.

Coronaviruses are enveloped viruses containing single-stranded RNA of positive polarity, belong to the Nidovirales order, the Coronaviridae family, which includes 2 subfamilies - Orthocoronavirinae and Letovirinae. The subfamily Orthocoronavirinae includes four genera: Alphacoronavirus, Betacoronavirus, Gammacoronavirus, Deltacoronavirus. As a rule, $\alpha$ - and $\beta$-coronaviruses infect mammals, and $\gamma$ - and $\delta$-coronaviruses infect birds [2].

\section{Goal of the Study}

The analysis of scientific literature data on the coronavirus disease pandemic of 2019. To achieve this goal, tasks were set to systematize, summarize and compare documentary data regarding historical aspects of coronaviruses; severe acute respiratory syndrome (SARS); Middle Eastern Respiratory Syndrome (MERS); SARS-CoV-2 virus disease COVID-19; comparative characteristics of SARS/MERS/COVID-19.

\section{Methods}

When conducting the study, we used the current legislative, regulatory and instructive documents; sources of scientific literature; Internet resources. We applied regulatory, documentary, bibliographic, systemic, comparative and graphical methods of analysis.

\section{Results and Discussion}

The history of the study of coronaviruses began in 1931, when the American veterinarians A.F. Schalk and M.C. Hawn described a "new respiratory disease" in chickens, in Russian-language literature called "chicken infectious bronchitis" [3].

The etiological agent of this disease, formerly called the Infectious bronchitis virus (IBV) virus, is now called avian coronavirus (ACoV, Gammacoronavirus, Igacovirus) and includes the previously independent turkey coronavirus (TCoV) and pheasant coronavirus (PhCoV). In 1946, American researchers L.P. Doyle and L.M. Hutchings described the pig transmissible gastroenteritis virus (TGEV). Later in 1986, the related porcine respiratory coronavirus (PRCV - Porcine respiratory coronavirus) was first described, followed by similar results for various animal species. In 1949, the mouse hepatitis virus (MHV) was described, which since 2011 has the modern name - mouse coronavirus (MCoV) [4].

Like many other coronaviruses, numerous MHV strains were divided into two patotypes, called enterotropic (causing cytolysis of enterocytes and numerous necrosis of the intestinal mucosa) and polytropic (reproducing in the epithelium of the nasopharynx, affecting the lymph nodes, but not the intestinal epithelium; [5] neurotropic strains are known).

The coronation of coronaviruses took place on $11 / 16 / 1968$, when the next $5168^{\text {th }}$ issue of the journal Nature was published, in which a group of eight virologists (including some authors of the first epidemic coronaviruses) published a short taxonomic proposal: due to the characteristic morphology of virons IBV, MHV, B814, 229E, OC43 in negatively contrasted electronic photographs - pronounced dentate (corona-shaped) framing (about $20 \mathrm{~nm}$ ) of rounded pleiomorphic particles (120 - $160 \mathrm{~nm})$ - combine these viruses into the coronaviruses group [6].

Already a year later, when describing RtCoV J.C. Parker., et al. (1970) used the term "coronavirus" in the title of the article. 
The coronavirus family includes two subfamilies. In the subfamily of orthocoronaviruses, four main genera distinguished alpha, beta, gamma and deltacoronaviruses. The first two genera primarily infect mammals, while the last two predominantly infect birds. Previously, before the outbreak of pneumonia at the end of 2019, six types of coronaviruses were identified as causing human disease. These include Human coronavirus 229E, Human coronavirus NL63 ( $\alpha$-coronaviruses), Betacoronavirus 1, Human coronavirus HKU1, Middle East respiratory syndrome related coronavirus, Severe Acute Respiratory Syndrome-related coronavirus ( $\beta$-coronaviruses). The genera of alpha and beta-coronaviruses are mainly found in mammals, such as bats, rodents, civet, humans. Gamma and deltacoronaviruses are mainly found in birds. Coronaviruses have the largest genomes among virus RNAs (from 25 to 32 thousand nucleotides). The SARS-CoV-2 genome consists of 29,881 nucleotides.

In electron micrographs, SARSCoV-2 particles represented mainly by rounded or oval particles with a diameter of 60 to 140 nanometers. Viral particles have rather characteristic spikes, about 9 - $12 \mathrm{~nm}$ long and this gives the virions the appearance of a solar corona. It is this characteristic feature that served as the basis for the name of the family of these viruses - coronaviruses [7].

By the beginning of the XXI century about coronaviruses, an opinion has emerged as relevant veterinary pathogens, but not of particular danger to humans. The situation changed dramatically in 2002 when the severe acute respiratory syndrome-related coronavirus (SARS-CoV) virus was identified as the etiological agent of the epidemic in the southern provinces of China.

The next stage of the study was the study of severe acute respiratory syndrome (SARS), which is a viral respiratory disease, caused by the subspecies of the coronavirus Severe acute respiratory syndrome coronavirus (SARS-CoV) [8].

It is believed that this is a virus of animal origin, breaking the interspecific barrier and becoming pathogenic to humans. SARS$\mathrm{CoV}$ belongs to the subgroup $2 \mathrm{~b}$ of $\beta$-coronaviruses. Transmission routes from animals to humans or other animal species remain unclear. The first human cases of SARS-CoV infection occurred in Guangdong (PRC), in November 2002, but the pathogen was detected only three months later. The cause of the global outbreak was the accelerated spread of the virus in Hong Kong. The trans- mission of the virus from person to person occurred mainly by airborne droplets and contact routes. In addition, cases of infection by the fecal-oral route through infected objects and surfaces were noted. During the epidemic, nosocomial transmission of SARS-CoV was common [8].

In 2003, the virus spread to 29 countries in which 8098 cases were recorded, mortality was almost $10 \%$, and for patients over 60 years of age - more than $50 \%$. The WHO announced the end of the epidemic in July 2003; the latest cases of SARS were reported in January 2004 [9].

The entrance gate to SARS infection is the respiratory tract. The binding of the virus to the cells is mediated by the receptors of angiotensin converting enzyme-2 (ACE2), which are extensively present in the epithelial cells of the alveoli, trachea, bronchi, as well as the small intestine. The duration of the incubation period of the disease ranges from two to ten days, an average of 4.6 days [10].

Then, an increase in body temperature $\left(38^{\circ} \mathrm{C}\right.$ and above $)$ develops, accompanied by fever, dry cough, sore throat and chest, myalgia, and often diarrhea, vomiting, and abdominal pain. After a few days, pneumonia develops, which in about $25 \%$ of cases progresses rapidly, which can lead to fatal respiratory failure.

During the epidemic, November 1, 2002 - July 31, 2003, the mortality rate in China was $9.2 \%$ (685/7429), and around the world, taking into account 667 cases and 89 deaths in 29 countries outside of China, due to imported cases - 9.6\% (774/8096). Found that the infection of people came from the Himalayan civet (Paguma larvata).

Further study of the ecology of SARS-CoV revealed that this virus is a natural focal virus, the natural reservoir is bats (Chiroptera, Microchiroptera), which transmit the infection inapparently, but secrete the virus with saliva, urine and feces, infecting small mammals that are widely used in the countries of Southeast Asia for food and for the needs of eastern medicine. The SARS-CoV epidemic spurred the study of 29 coronaviruses. In 2004, Dutch researchers described human coronavirus NL63 (from English Sample number 63 from the Netherlands) (HCoV-NL63 - Human coronavirus NL63) (Alphacoronavirus). In January 2005, employees of Hong Kong University isolated from a 71-year-old patient with acute respiratory infections complicated by bilateral pneumonia the new hu- 
man coronavirus HKU1 (HCoV-HKU1 - Human coronavirus HKU1) (Betacoronavirus), initiating the use of the HKU prefix (from Hong Kong University) with the serial number of the strain, which is found in the names of many viruses [11].

The next stage of the study concerned the Middle East Respiratory Syndrome (MERS), which is a viral respiratory disease, caused by the Middle East respiratory syndrome-related coronavirus (MERS-CoV) coronavirus. The history of the study of this virus began in June 2012, when a viral isolate with a pronounced cytopathic effect was obtained on a model of transplanted cell lines from a nasopharyngeal washout of a 60-year-old man with community-acquired pneumonia, who later died in a hospital in Jeddah (western Saudi Arabia) African green monkey kidney (Vero) and rhesus macaque (LLC-MK-2). The polymerase chain reaction with universal coronavirus primers allowed the primary identification of the pathogen as a representative of Coronaviridae [12].

Subsequent sequencing was carried out at the University of Erasmus (Netherlands), where it was found that the new betacoronavirus is related but not identical to SARS-CoV, being the closest in genetic terms to BtCoV-HKU4 and BtCoV-HKU5. In September 2012, a 49-year-old man with community-acquired bilateral pneumonia was admitted to one of the London clinics. The patient was taken from the hospital of Qatar, before that he visited Saudi Arabia and died on the $30^{\text {th }}$ day from the onset of the disease. A strain of MERS-CoV was also isolated from the patient's tracheal aspirates. Retrospective serological studies showed that at least 9 cases of community-acquired pneumonia, of which 2 were fatal (confirmed by RT-PCR pathological material) among patients in Jordanian hospitals in March-April (12 - 24 $4^{\text {th }}$ week) 2012 were associated with MERS-CoV [13].

It became clear that humanity faced with a new particularly dangerous coronavirus. An analysis of the infectious dynamics of MERS-CoV allowed domestic specialists to make a forecast at the end of 2013 about an "increased level of epidemic danger", which coincided with the beginning of a large-scale epidemic outbreak in the spring - summer (14 - 24 $4^{\text {th }}$ week) of 2014 in Saudi Arabia with mortality rate of $42.1 \%(110 / 261)$. According to WHO data at the beginning of January 2020, mortality from MERS-CoV in 27 countries of the world amounted to $34.4 \%$ (866/2519) [14].

MERS-CoV, like SARS-CoV, is an animal coronavirus capable of infecting humans. The route of transmission from animals to hu- mans is not fully understood, but one-humped camels are probably an intermediate host for MERS-CoV and a source of infection for humans. People infected by direct or indirect contact with infected camels. The virus cannot be easily transmitted from person to person if there is no close contact, for example, with unprotected care of an infected patient. Human-to-human transmission is limited, and so far, only infections have been identified among family members, patients, and health workers. To date, no steady transmission of the virus from person to person has been recorded in the world. About $80 \%$ of human cases were reported in Saudi Arabia. Need to note that cases detected outside the Middle East were related to the movement of people infected in the Middle East. The primary receptor for MERS-CoV infection is the multifunctional surface cell protein dipeptidyl peptidase-4 (DPP4), expressed in large quantities on the epithelial cells of the kidneys, alveoli, small intestine, liver and prostate. On average, the incubation period lasts 5 - 6 days, varying from two to sixteen days [15].

Clinical manifestations and their severity can vary greatly: from the absence of symptoms (asymptomatic) or mild respiratory manifestations to severe acute pulmonary diseases and death. A typical manifestation of the disease is fever, cough, and shortness of breath. Pneumonia is a common complication, but is not always present. Gastrointestinal upset symptoms, including diarrhea, are also noted. Severe illness can cause respiratory failure, which requires mechanical ventilation and the patient is in the intensive care unit. The virus causes more severe diseases in the elderly, people with weakened immune systems and people with chronic diseases such as kidney failure, cancer, chronic lung diseases and diabetes [16].

Subsequently, a study was conducted of the disease COVID-19 caused by the SARS-CoV-2 virus. On December 31, 2019, the Chinese health authorities informed WHO of cases of pneumonia of unknown etiology found in Wuhan (Hubei Province, PRC). On January 7, 2020, experts from China found that the cause is coronavirus 2019-nCoV (SARS-CoV-2). On February 11, 2020, WHO officially named the disease caused by the SARS-CoV-2 virus [17] as the coronavirus disease 2019 (COVID-19 or COVIB-19). On the same day, the coronavirus research group of the International Committee on the Taxonomy of the Virus, previously named 2019-nCoV, renamed the pathogen SARS-CoV-2 (Severe acute respiratory syndrome-related coronavirus- 2 or severe acute respiratory syndrome coronavirus-2). Epidemiological studies have found that 
most cases of infection were associated with the wholesale market of the city, which they visited before the disease, where various seafood was sold, and also traded in many species of live and killed domestic and wild animals (poultry, bats, marmots, snakes, frogs, hedgehogs, rabbits, etc).

SARS-CoV-2 coronavirus is a new strain that has not previously been identified in humans. On January 30, 2020, the WHO Emergency Committee announced that the current outbreak of coronavirus infection met international emergency health criteria. As in the case of SARS-CoV and MERS-CoV, bats can be the main reservoir of SARS-CoV-2 infection, but no intermediate host has been identified. The eleven millionth city of Wuhan is the capital of one of the most (60 million people) and densely populated (323 people/ $\mathrm{km}^{2}$ ) Chinese provinces with a developed transport system, so the epidemic developed rapidly in the epicenter (in Hubei province) and was accompanied by a large number of imported cases both in other provinces of China and in other countries. By January 30 , 2020, the WHO Emergency Committee had declared an epidemic outbreak in Hubei caused by $2019-n C o V$ as a public health emergency of international concern: the global mortality rate was $2.2 \%$ (170/7818); outside of China, $0.0 \%(0 / 82)$ in 18 countries [18].

Phylogenetic analysis of fragments of various genes of the SARS-CoV-2 virus and analysis of the complete genome of this virus, isolated from a number of patients, showed that this virus is a new beta-coronavirus (subfamily of Orthocoronaviruses) belonging to line B or the sarbekovirus subgenus, which also includes human SARS coronavirus. The genomes of the viral strains analyzed are phylogenetically closest to coronaviruses associated with a disease like SARS in bats (Rhinolophus sinicus), first isolated from them in Zhoushan, Zhejiang Province, China, from 2015 to 2017. It was found that the complete genome of the SARS-CoV-2 virus has approximately $89 \%$ nucleotide identity with bat-SL-CoVZC45, which makes it a new species. Moreover, the spike protein genome of this virus has 84 - 88\% nucleotide identity with bat-SL-CoVZC45 coronavirus and $78-79 \%$ nucleotide identity with human SARS coronavirus and 50\% with MERS-Cov [19].

Molecular analysis of the available viruses also found that the common ancestor of the SARS-CoV-2 virus appeared around late September - early December (on average in November) 2019. It has been suggested that the SARS-CoV-2 virus appears to be a recombinant coronavirus between coronaviruses that cause SARS- like diseases in bats and an unknown coronavirus. It must be pointed out that the dynamics of the increase in incidence cases, as well as the epidemiological characteristics of the outbreak, also indicate the possibility of transmission of infection from person to person in a variety of ways: airborne, contact. It is believed that the leading is the airborne transmission mechanism. The infection of health workers in caring for infected patients and the first deaths among them are documented. It should be noted that the virus is also detected in feces and the possibility of fecal-oral transmission of the infection is not excluded. This transmission mechanism is very likely, especially if one takes into account the high expression of ACE2 molecules on enterocytes and large amounts of virus (exceeding that in the blood) found in anal scrapings in individual patients. It is important that high expression of the ACE2 molecule was found on epithelial cells of the human oral cavity. In this case, it is necessary to take into account the revealed features of the virus envelope, which, as suggested by the authors, indicate greater virus stability in the external environment, which contributes to the possibility of SARS-Cov-2 transmission not only by the respiratory, but also by the fecal-oral route [20].

The results of recent studies indicate a rather long period of SARS-Cov-2 isolation and its detection in smear and sputum samples with a peak of isolation (approximately $10^{4}$ to $10^{4}$ copies $/ \mathrm{ml}$ ) approximately 5 - 6 days after the onset of symptoms. Moreover, the level of virus in sputum, as a rule, exceeded its level in swabs from the oropharynx and a high degree of correlation was observed between paired samples. The virus is constantly detected in the blood. Therefore, theoretically, there is a risk of transmission of coronaviruses during transfusion of blood products. As more and more people with asymptomatic infection are found among COVID-19 cases, ensuring the safety of blood recipients with respect to the SARS, MERS, SARS-CoV-2 coronaviruses is relevant, especially in endemic areas. When analyzing the SARS-Cov-2 epidemic, WHO specialists found that the base reproductive number (that is, the average number of people infected from one patient) ranges from 1.4 to 2.5 , which is less than the same indices for acute respiratory viral infections ( 2 to 5 ). However, it should be borne in mind that this number may increase if the epidemic is not controlled by quarantine and isolation [21].

It should be noted that there is a significant variation in this indicator of the intensity of infection transmission in the studies, which, apparently, depends on many factors. This underlines the 
importance of surveillance in countries around the world to ensure that the ongoing outbreak does not become a global pandemic.

When modeling the SARS-CoV-2 virus thorn protein, it was concluded that the molecule of angiotensin-converting enzyme 2 (ACE2) is a receptor for this virus. This molecule is known to be a receptor for SARS coronavirus. According to this model, the strength of the interaction between the spike molecule ( $\mathrm{S}$ molecule) of SARS-CoV-2 and ACE2 is higher than the threshold required for the occurrence of viral infection, although weaker than between SARS [22].

It was found that $2.5 \%$ to $5.6 \%$ of non-immune cells of the nasal mucosa, oral cavity, lungs and colon epithelial cells have ACE2 expression with a tendency to coexpression with the HLA-DRB1 molecule. However, to date, there is evidence showing that Asian donors have about five times more cells expressing ACE2 than donors, Caucasian and African Americans. The disproportionately high number of people infected with the virus of the Asian (Mongoloid) race in such an international center as Shanghai is also consistent with the above data. These results indicate a possible increased susceptibility of the Asian population to the SARS-CoV-2 virus, although, of course, they require more evidence to draw conclusions. There is also evidence that men have a higher level of ACE2 expression on alveolar cells than women. Studies have shown that ACE2 is highly expressed by a wide range of human body cells (cells of the proximal tubule of the kidneys, enterocytes, etc.) and characterize possible pathogenetic mechanisms of the development of lesions in SARS-CoV-2 infections outside the lungs. It is likely that liver damage and an increase in the level of ALT, AST, and other enzymes that correlate with the severity of coronavirus disease 2019 are explained by the peculiarities of ACE2 expression by liver cells [23].

At the last stage of this study, a comparative characteristic of SARS/MERS/COVID-19 was given by: the source of the disease; the mechanism of transmission from animal to human; human-tohuman transmission mechanism; epidemiology; distribution by gender and age; concomitant diseases; targeted populations [24].

he source of the disease

For all three viruses, bats are the likely primary natural reservoir of infection. The transmission of the virus to humans occurred, presumably, through an intermediate host: civet (SARS-
$\mathrm{CoV}$ ), one-humped camels (MERS-CoV) or a definitively unknown specific animal species, possibly civet, pangolins or directly from bats, which is less likely (SARS-CoV-2).

The mechanism of transmission from animal to human

The mechanism and transmission routes of coronaviruses remain unknown. For SARS-CoV and MERS-CoV, the main routes of transmission may be direct contact with an infected animal (intermediate host) or the ingestion of milk, non-processed meat or contact with the urine (MERS) of infected animals.

For SARS-CoV-2, direct contact, eating poorly processed meat, or using parts of the body of infected animals as a component in the therapeutic agent of traditional Chinese medicine is also assumed.

\section{The mechanism of transmission from person to person}

The primary transmission route from person to person for MERS-CoV is via contact; for SARS-CoV and SARS-CoV-2 - airborne and contact. Nosocomial transmission has played a major role in the spread of SARS and MERS and is significant during the COVID-19 epidemic.

\section{Epidemiology}

The current outbreak of COVID-19 significantly exceeds previous SARS and MERS outbreaks in the total number of reported cases, while the mortality rate among patients $(3.4 \%$ as of $02 / 27 / 2020)$ is significantly lower than in previous epidemic outbreaks. Over the period of this epidemic outbreak, more than 2 thousand people have already died, which exceeds the total number of deaths during the SARS and CoV epidemics. Need to note the existing cases of the asymptomatic course of COVID-19 (about 1.2\%); however, the asymptomatic course was also found in SARS and MERS, but much less frequently. COVID-19 is characterized by a generally milder course of the disease than with SARS and MERS.

\section{Distribution by gender and age}

According to a study by The Novel Coronavirus Pneumonia Emergency Response Epidemiology Team (NCPERET), the SARCCoV-2 virus infects both men and women almost equally (the ratio of male and female patients is 1.06: 1), while mortality among sick men is significantly higher. The distribution of cases by sex for SARS (0.75: 1, respectively) and MERS differs from COVID-19, which is probably due to a significantly smaller number of analyzed cases, and, in the case of MERS, where there is almost 2 times more cases 
of the disease in men than in women can be explained by regional cultural characteristics (the dominant position of men in society). Almost 78\% of cases of COVID-19 were found in people aged 30 to 69 years inclusive; mortality among patients increases with age.

\section{Accompanying illnesses}

Concomitant diseases are a serious risk factor for more severe coronavirus pneumonia and death. For SARS, chronic heart disease, diabetes, chronic hepatitis B, malignant neoplasms, and chronic pulmonary diseases were major adverse prognostic factors. In the case of MERS, such factors are diabetes, hypertension, chronic renal failure, malignant neoplasms, chronic diseases of the heart, lungs and liver. For COVID-19, concomitant diseases that worsen the course of the disease and its outcome include cardiovascular disease, diabetes, chronic respiratory disease, hypertension, and cancer.

\section{Target groups}

Persons with concomitant diseases, smokers, health workers (COVID-19, MERS, SARS), elderly people (COVID-19, MERS), obese people (MERS), etc. are at increased risk of infection.

Thus, by the end of April 2020 there are still many questions about the new coronavirus: its natural reservoir, transmission routes, and pandemic potential. Based on the rapid spread of infection, a large number of infected people and a high baseline rate of reproduction of the infection, it can be argued that the current COVID-19 epidemic is more dangerous compared to previous outbreaks of pneumonia caused by coronaviruses. The high level of threat to the global spread of the new virus is recognized by the WHO, as vaccines and antiviral drugs against coronaviruses have not yet been developed, and knowledge of their epidemiology is limited.

The above indicates the need to continue further research and reviews of the coronavirus pandemic in the direction of finding methods for effective treatment of patients with this pathology.

\section{Conclusion}

- The analysis of scientific literature data on the coronavirus pandemic of 2019 was performed.

- The historical aspects of the study of coronaviruses were studied.
- Systematized and summarized documentary data regarding severe acute respiratory syndrome (SARS); Middle Eastern Respiratory Syndrome (MERS); SARS-CoV-2 virus disease COVID-19.

- The comparative characteristic of SARS/MERS/COVID-19 is given.

- The need for further study of the coronavirus pandemic towards pharmacotherapy.

\section{Bibliography}

1. Chen Y and Guo D. "Molecular mechanisms of coronavirus RNA capping and methylation". Virologica Sinica 31.3 (2016): 3-11.

2. Woo PCY., et al. "Discovery of seven novel mammalian and avian coronaviruses in the genus deltacoronavirus supports bat coronaviruses as the gene source of alphacoronavirus and betacoronavirus and avian coronaviruses as the gene source of gammacoronavirus and deltacoronavirus". Journal of Virology 86 (2012): 3995-4008.

3. Schalk AF and Hawn MC. "An apparently new respiratory disease of baby chicks". Journal of the American Veterinary Medical Association 78 (1931): 19.

4. "Virus Taxonomy. Classification and Nomenclature of Viruses. Ninth Report of the International Committee on Taxonomy of Viruses". Eds. King A. M. Q., Adams M. J., Carstens E. B., Lefkowitz E. J. Elsevier Academic Press (2011): 1338.

5. Baker DG. "Natural pathogens of laboratory mice, rats, and rabbits and their effects on research". Clinical Microbiology Reviews 11 (1998): 231-266.

6. Almeida JD., et al. “Virology: Coronaviruses”. Nature 220 (1968).

7. Li F. "Structure, Function, and Evolution of Coronavirus Spike Proteins". Annual Reviews of Virology 29 (2016): 237-261.

8. Peiris JS., et al. "Coronavirus as a possible cause of severe acute respiratory syndrome”. Lancet 361.9366 (2003): 1319-1325.

9. Hui DS and Zumla A. "Severe acute respiratory syndrome. Historical, epidemiologic, and clinical features". Infectious Disease Clinics of North America 33.4 (2019): 869-889. 
10. Donnelly CA., et al. "Epidemiological determinants of spread of causal agent of severe acute respiratory syndrome in Hong Kong". Lancet 361.9371 (2003): 1761-1766.

11. Virology Guide. "Viruses and viral infections of humans and animals". Ed. Lvov D.K. M.: MIA (2013): 1200.

12. Zaki AM., et al. "Isolation of a novel coronavirus from a man with pneumonia in Saudi Arabia". The New England Journal of Medicine 367.19 (2012): 1814-1820.

13. Al-Abdallat MM., et al. "Hospital associated outbreak of Middle East respiratory syndrome coronavirus: a serologic, epidemiologic, and clinical description". Clinical Infection Disease 59 (2014): 1225-1233.

14. World Health Organization. MERS situation update (2020).

15. Mackay IM., et al. "MERS coronavirus: diagnostics, epidemiology and transmission". Virology Journal 12 (2015): 222.

16. Yin $Y$ and Wunderink RG. "MERS, SARS and other coronaviruses as causes of pneumonia". Respirology 23.2 (2018): 130-137.

17. Cui J., et al. "Origin and evolution of pathogenic coronaviruses". Nature Review on Microbiology 17 (2019): 181-192.

18. World Health Organization. Novel Coronavirus (2019-nCoV). Situation Report-10 (2020).

19. JF Jhan., et al. "A familial cluster of pneumonia associated with the 2019 novel coronavirus indicating person-to-person transmission: a study of a family cluster". Lancet 395 (2020): 514523.

20. GK Goh., et al. "Rigidity of the Outer Shell Predicted by a Protein Intrinsic Disorder Model Sheds Light on the COVID-19 (Wuhan-SARS-CoV-2) Infectivity". Biomolecules 10 (2020).

21. D Benvenuto., et al. "The global spread of 2019-nCoV: a molecular evolutionary analysis". Pathology Global Health 12 (2020): $1-4$.

22. X Xu., et al. "Evolution of the novel coronavirus from the ongoing Wuhan outbreak and modeling of its spike protein for risk of human transmission". Life Science (2020).

23. GW Guan., et al. "Exploring the mechanism of liver enzyme abnormalities in patients with novel coronavirus-infected pneu- monia". Zhonghua Gan Zang Bing Za Zhi = Zhonghua Ganzangbing Zazhi = Chinese Journal of Hepatology 28 (2020).

24. DV Gorenkov., et al. "Outbreak of a new infectious disease COVID-19: $\beta$-coronaviruses as a threat to global health". Biopreparations. Prevention, Diagnosis, Treatment 20 (2020): 6-20.

\section{Assets from publication with us}

- Prompt Acknowledgement after receiving the article

- Thorough Double blinded peer review

- Rapid Publication

- Issue of Publication Certificate

- High visibility of your Published work

Website: www.actascientific.com/

Submit Article: www.actascientific.com/submission.php Email us: editor@actascientific.com

Contact us: +919182824667 